\title{
ORIGINAL ARTICLE \\ Comparison of gait between healthy participants and persons with spinal cord injury when using a powered gait orthosis-a pilot study
}

\author{
M Arazpour ${ }^{1}$, SR Mehrpour ${ }^{2}$, MA Bani ${ }^{1}$, SW Hutchins ${ }^{3}$, M Bahramizadeh ${ }^{1}$ and M Rahgozar ${ }^{4}$
}

Objectives: The aim of this study was to evaluate the effect of a powered gait orthosis (PGO) on the temporal-spatial parameters and kinematics of walking in both healthy participants and persons with spinal cord injury (SCI) using three-dimensional motion analysis to facilitate further development of such devices.

Methods: Kinematics and temporal spatial data were obtained from three healthy participants and four persons with $\mathrm{SCl}$ who walked using the same design of PGO.

Results: Walking speed was reduced by $28 \%$ and step length by $29 \%$ in healthy individuals when walking with PGO compared with normal walking and that recorded for persons with $\mathrm{SCl}$ was approximately one-third that of normal walking. There were significant differences in hip and knee joint ranges of motion in comparison between walking with PGO in healthy participants and walking with $\mathrm{PGO}$ in persons with $\mathrm{SCl}$.

Conclusion: Walking with a PGO by healthy participants significantly reduced critical gait parameters, and further development work is needed to produce a more effective device to match closely the gait parameters of normal walking by healthy participants. Significant differences between normal walking and that evidenced with the PGO by both healthy participants and persons with $\mathrm{SCl}$ were detected.

Spinal Cord (2014) 52, 44-48; doi:10.1038/sc.2013.139; published online 3 December 2013

Keywords: spinal cord injury; paraplegia; powered gait orthosis; gait analysis; walking; optimal level of walking

\section{INTRODUCTION}

Many treatments have been developed for patients with spinal cord injury (SCI) to help them walk. One such development is the use of powered gait orthoses (PGOs), which utilize externally powered actuators using pneumatics, hydraulics or electrical power to apply power to the hip, knee and, in some designs, ankle joints in such orthoses. ${ }^{1}$

PGOs reduce the effort required to walk when using the upper limb muscles for support and propulsion, and also provide a means of static support for upright posture. ${ }^{2}$ High loads on the upper limb joints and high levels of energy consumption have been demonstrated when walking with non-powered mechanical orthosis by persons with SCI. ${ }^{3,4}$ These types of orthosis have therefore been developed to improve the efficiency of walking compared with that offered by passive orthoses such as the advanced reciprocating gait orthosis), the isocentric reciprocating gait orthosis (IRGO), the Prime walk and the Walkabout orthosis on walking for paraplegic patients. One of the disadvantages of ambulating with standard non-powered RGOs is that the mean hip flexion range of motion (ROM) on one side is directly related to the amount and rate of extension in the opposite side owing to the direct mechanical link between the two sides in the orthosis. This restriction limits and reduces the speed of walking and step length during ambulation. ${ }^{5,6}$ Walking and forward movement is achieved by use of the upper limbs and contraction of trunk muscles in persons with SCI, which leads to a high energy expenditure when ambulating, ${ }^{3}$ and can lead to premature exhaustion. ${ }^{3,7}$

The positive effects of powered orthoses on the gait parameters of persons with SCI have therefore been demonstrated in the literature. ${ }^{1}$ However, these improvements differ in patients according to their level of injury, ${ }^{8}$ and the optimal injury level of walking with powered orthosis is not clear. Healthy participants, when walking with a powered orthosis, may also demonstrate abnormal gait patterns. Further knowledge of how 'normal' walking can be when healthy participants walk with a powered orthosis is needed to determine the limitations involved. It may transpire that using a powered orthosis has specific effects on healthy participants walking. It would therefore be useful to quantify these in order to optimize the level of walking and operational parameters needed when powered orthosis are used for persons with SCI. This is so that they may ambulate within parameters designed into the orthosis mechanism that are appropriate for their condition and injury level.

There is limited evidence available in the literature when comparing walking in powered gait orthosis (PGOs) between healthy individuals and persons with SCI. Only one study was found, which

${ }^{1}$ Orthotics and Prosthetics Department, University of Social Welfare and Rehabilitation Sciences, Tehran, Iran; ${ }^{2}$ Orthopedic surgeon, Shariati Hospital, Tehran University of medical Science, Tehran, Iran; ${ }^{3}$ University of Salford, IHSCR, Faculty of Health \& Social Care, University of Salford, Salford, UK and ${ }^{4}$ Biostatistical Department, University of Social Welfare and Rehabilitation Sciences, Tehran, Iran

Correspondence: MA Bani, Department of Orthotics and Prosthetics, University of Social Welfare and Rehabilitation Sciences, Kodakyar St., Daneshjo Blvd., Evin, Tehran 1985713834, Iran.

E-mail: m.ahmadi_bani@yahoo.com

Received 16 May 2013; revised 13 October 2013; accepted 16 October 2013; published online 3 December 2013 
evaluated the interaction between healthy participants when walking with a newly developed RGO with a variable constraint hip mechanism to determine their restrictions and their impact on functional performance. ${ }^{9}$ To the authors' knowledge, no evidence exists using gait analysis as to the impact of a powered orthosis on the temporalspatial parameters and kinematics of walking when worn by both healthy participants and persons with SCI while participating in a comparative study using the same orthosis. Data from the study would be expected to help professionals hypothesize improvements in the design of powered orthoses and in the application of them for persons with SCI. Therefore, the purpose of this study was to compare the use of PGO between healthy individuals and persons with SCI on the temporal-spatial parameters and kinematics of walking. The primary outcome measures were chosen to enable a direct comparison to be made between the differences between them, demonstrated by healthy individuals and persons with SCI to define operational parameters and limits of joint motion required in a powered orthosis.

\section{METHODS}

\section{Subjects and experimental protocol}

Three healthy participants (Table 1) and four persons with SCI (Table 2) took part in the study. All healthy participants were able-bodied with no known history of orthopedic illness or any other ailment that would have an effect on their walking pattern. The persons with SCI were all experienced orthotic users with normal upper limb strength. Exclusion criteria for persons with SCI were presence of cardiovascular or pulmonary illness, contractures, severe spasticity, obesity or asymmetric hip positions. The four persons with SCI all had motor complete impairments (AIS grades A or B) and therefore were not able to ambulate without using an orthosis of some type. Each person with SCI and healthy participants were fitted with a PGO, the design and simulation of which has been reported previously. ${ }^{10}$ The effect of walking with the orthosis had been previously evaluated on a patient with T8 spinal cord injury. ${ }^{11}$ Written consent was obtained from each healthy participants and persons with SCI before being included in the study. The experimental protocol for the study was approved by the ethical committee of University of Social Welfare and Rehabilitation Sciences.

The participants walked along a $6 \mathrm{~m}$ walkway in a gait laboratory. The gait evaluation system consisted of a 6 Camera Vicon digital motion capture system (Oxford Metrics, Oxford, UK). The standard Plug-in Gait marker set was used. A total of 18 reflective markers were placed on the lower extremities and the trunk over the position of the greater trochanters, the lateral condyles of the femur, bilaterally on the fibular head and lateral malleolus, the second metatarsals, both anterior superior iliac spine and calcaneus, and over the jugular notch, plus on the spinous process of the seventh cervical vertebrae and the acromioclavicular joints.

During normal walking, markers were placed on the subject's skin, but when walking with the orthosis, the markers of lower extremity were put on the uprights of the orthosis as close as possible to the anatomical landmark positions. ${ }^{12}$ ROM of the hip, knee and ankle joint were calculated by measuring these angles between reference frames defined on the trunk, thigh, shank and foot segments. The markers put on the body and orthosis segments were used to define the frames.

Table 1 Healthy subject demographics ( \pm s.d.)

\begin{tabular}{lc}
\hline Parameter & Value \\
\hline Number of subjects & 3 \\
Gender & 3 Male \\
Age (years) & $29 \pm 1.00$ \\
Height (cm) & $172 \pm 2.51$ \\
Weight (kg) & $62.33 \pm 2.51$ \\
\hline
\end{tabular}

For all walking trials with and without PGO, the healthy participants walked with a walker as would persons with SCI. In this condition, uniformity of the data between two groups and stability of participants were provided in this study. For each of the test conditions, healthy participants and persons with SCI walked along the walkway five times. This ensured that approximately 30 gait cycles were considered and averaged for each condition. Healthy participants walked both with and without the orthosis. In all conditions when walking with the PGO, the knees and hip joints were actuated to provide synchronized movements. The ankles were fixed using solid ankle foot orthoses bilaterally. Each healthy participants and persons with SCI also undertook five practice walks along the walkway to adapt to the orthosis before walking data were captured.

\section{Orthoses and orthotic configurations}

A PGO was used in this study. Design and simulation of this PGO has previously been reported on walking in three healthy subjects. Arazpour et al. ${ }^{10}$ demonstrated that this PGO could be used to assist persons with SCI and participants with impaired lower limb function (e.g. stroke, poliomyelitis, myelomeningocele and traumatic brain injury) who have adequate ranges of motion to ambulate. In other study, Arazpour et al. ${ }^{11}$ analyzed the effect of using this PGO on walking in one spinal cord injury participant and reported that this newly developed orthosis showed improvement in speed of walking, step length, maximum hip flexion and extension angles and maximum knee flexion and extension angles when compared with walking with an IRGO. The results from this case study gave the authors confidence to extend the research to a group of people with spinal cord injury.

The orthosis was a prototype IRGO equipped with actuated hip and knee joints, which was designed to assist standing and facilitate walking for individuals with paraplegia. ${ }^{10}$ The orthosis was developed to provide upright posture and walking for patients with SCI, and utilized finite-state robot-based electrically actuated motors attached to the orthosis to perform active flexion and extension of the hip and knee joints. The electronics and motor included a Maxon Motor EC30 (Maxon, Sachseln, Switzerland) with a planetary gearbox with reduction of 156 for the hip joints and Maxon Motor EC45 (Maxon) with a planetary gearbox with a reduction of 110 for the knee joints. The system also included encoders, motor drivers, a power supply and a programmable controller. The motor sequencing was based on models optimized by software specifically designed and built for this purpose. The orthotic joint motion patterns could then be programmed into the microcontroller to the orthosis. The batteries and control board were placed within a backpack that was worn by subject during orthosis-using time. The operation of the PGO during walking was initiated by the orthotist via a joystick. ${ }^{10,12}$

The patient transferred weight to the left foot, and the right-side knee actuator was then activated, which flexed knee joint followed by the ipsilateral hip joint actuator, which flexed the hip joint. The patient then moved the pelvis forward on the same side. The right-side knee actuator was activated again to provide extension of the knee joint, after which the hip joint actuator was activated and hip joint extended. This performance permitted the patient to transfer her/him weight to the right leg. This cycle was then repeated for the left foot. During all these process, the left knee joint actuator locked the leftside knee joint in extension.

The healthy participants had 1 week of gait training education with the new orthosis in an outpatient orthotic clinic and the SCI patients performed gait training with the PGO for a minimum of 6 weeks, at $1 \mathrm{~h}$ per day for 5 days per week before gait evaluation. After the gait training period with

\section{Table 2 Characteristics of the persons with $\mathrm{SCl}$}

\begin{tabular}{llcccccc}
\hline Patient Sex & $\begin{array}{c}\text { Age } \\
\text { (years) }\end{array}$ & $\begin{array}{c}\text { Height } \\
\text { (cm) }\end{array}$ & $\begin{array}{c}\text { Weight } \\
\text { (kg) }\end{array}$ & $\begin{array}{c}\text { Level of } \\
\text { injury }\end{array}$ & $\begin{array}{c}\text { ASIA } \\
\text { score }\end{array}$ & $\begin{array}{c}\text { Time since injury } \\
\text { (months) }\end{array}$ \\
\hline 1 & Male & 29.0 & 165.0 & 53.0 & T12 & B & 32.0 \\
2 & Female & 22.0 & 165.0 & 54.0 & T8 & B & 51.0 \\
3 & Male & 26.0 & 178.0 & 65.0 & T10 & A & 15.0 \\
4 & Female & 29.0 & 160.0 & 59.0 & T6 & B & 9.0 \\
\hline
\end{tabular}

Abbreviations: ASIA, American Spinal Injury Association; SCI, spinal cord injury. 
the new PGO, each patient could walk continuously and independently with the orthosis.

The uprights of new orthosis were constructed to be adjustable so that this orthosis could be used by all the healthy and SCI volunteer participants. The polypropylene solid ankle foot orthoses were, however, custom made for each participant. The new orthosis weighed approximately $10 \mathrm{~kg}$. Kinematic and temporal spatial (speed of walking, step length, cadence) data were calculated under three conditions: (1) normal walking without an orthosis (healthy participants only); (2) walking with the PGO by healthy participants; and (3) walking with the PGO by the persons with SCI. Figure 1 shows walking with PGO in this study.

\section{Data analysis}

To assess the effect of the two walking conditions with the orthosis and following the results in normal walking without the orthosis, one-way analysis of variances were used. Tukey's multiple comparisons were performed when the

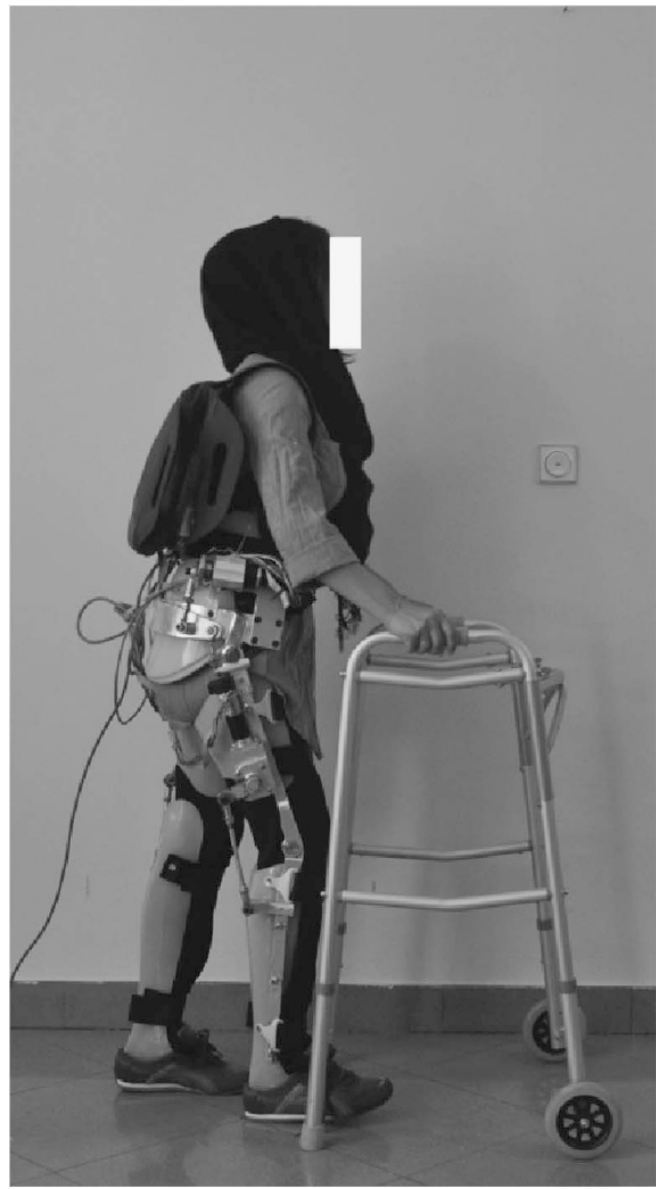

Figure 1 Participant walking with the PGO. analysis of variance was significant. SPSS version 16.0 (SPSS Inc., Chicago, IL, USA) was used for data analysis. The significant level considered was $\alpha=0.05$.

\section{RESULTS}

Table 3 shows the means \pm s.d.'s of the primary outcome measures and Table 4 shows the relevant $P$-values when comparing the three test conditions. The self-selected walking speeds and step lengths were both significantly reduced by $28 \%(P=0.006)$ and $29 \%(P=0.007)$, respectively, in healthy participants compared with normal walking when walking with the PGO, but cadence was increased. This coincided with reduced hip ROM $\left(5^{\circ}\right)$ and reduced knee ROM $\left(25^{\circ}\right)$ compared with normal. Although there was no significant difference between normal walking and walking with PGO in healthy participants in hip joint ROM $(P=0.081)$, but there was significant difference between normal walking and walking with PGO in healthy participants in knee joint $\mathrm{ROM}(P=0.000)$.

Walking speed with the new PGO in persons with SCI was only about one-third of that typically obtained in walking without the orthosis in healthy participants and was significantly reduced $(P=0.000)$. It was also significantly less compared with walking in the PGO by healthy participants $(P=0.001)$. Hip and knee ROMs were both significantly reduced ( $P=0.003$ and 0.041 , respectively) in comparison between walking with PGO in healthy participants and walking with PGO in persons with SCI.

Ankle joint sagittal plane ROM was reduced by the PGO in healthy participants compared with their normal walking because of the action of the ankle foot orthosis sections of the orthosis.

\section{DISCUSSION}

The results demonstrated that walking with the PGO produced a lower walking speed in both participant groups when compared with normal walking. This was less than half the speed achieved by healthy participants when the PGO was used by persons with SCI. Interestingly, cadence was increased by healthy participants when walking with the orthosis compared with normal, but that for persons with SCI was significantly reduced by approximately $45 \%$ compared with

Table $4 P$-values of hip, knee and ankle joint ROM and spatialtemporal gait parameters between the three test conditions

\begin{tabular}{lcccccc}
\hline $\begin{array}{l}\text { P- } \\
\text { value }\end{array}$ & $\begin{array}{c}\text { Speed of } \\
\text { walking }\end{array}$ & Cadence & $\begin{array}{c}\text { Step } \\
\text { length }\end{array}$ & $\begin{array}{c}\text { Hip } \\
\text { ROM }\end{array}$ & $\begin{array}{c}\text { Knee } \\
\text { ROM }\end{array}$ & $\begin{array}{c}\text { Ankle } \\
\text { ROM }\end{array}$ \\
\hline P1 & 0.006 & 0.369 & 0.007 & 0.081 & 0.000 & 0.000 \\
$P 2$ & 0.000 & 0.016 & 0.001 & 0.000 & 0.000 & 0.000 \\
$P 3$ & 0.001 & 0.003 & 0.269 & 0.003 & 0.041 & 0.095
\end{tabular}

Abbreviations: PGO, powered gait orthosis; ROM, range of motion; $\mathrm{SCl}$, spinal cord injury. $P 1$ : Comparison between normal walking and walking with PGO in healthy participants. $P 2$ : Comparison between normal walking and walking with $\mathrm{PGO}$ in persons with $\mathrm{SCI}$. $P 3$ : Comparison between walking with PGO in healthy participants and walking with PGO in persons with $\mathrm{SCl}$.

Table 3 Mean \pm s.d. of hip, knee and ankle joints ROM and spatial-temporal gait parameters in walking with PGO in persons with SCl and normal walking with and without orthosis

\begin{tabular}{|c|c|c|c|c|c|c|}
\hline & Speed of walking $\left(\mathrm{ms}^{-1}\right)$ & Cadence (steps per min) & Step length $(\mathrm{cm})$ & Hip ROM (deg.) & Knee ROM (deg.) & Ankle ROM (deg.) \\
\hline Normal walking without orthosis & $1.22 \pm 0.09$ & $90 \pm 2$ & $62.66 \pm 2.51$ & $41.44 \pm 1.23$ & $66.86 \pm 1.56$ & $39.03 \pm 1.33$ \\
\hline Walking with PGO in healthy participants & $0.87 \pm 0.04$ & $106 \pm 10$ & $49.00 \pm 2.64$ & $36.33 \pm 1.52$ & $41.33 \pm 2.08$ & $8.66 \pm 1.02$ \\
\hline Walking with PGO in persons with $\mathrm{SCl}$ & $0.40 \pm 0.11$ & $49 \pm 19$ & $44.15 \pm 4.85$ & $26.50 \pm 3.31$ & $37.00 \pm 1.82$ & $10.77 \pm 1.00$ \\
\hline
\end{tabular}

Abbreviations: PGO, powered gait orthosis; ROM, range of motion; $\mathrm{SCl}$, spinal cord injury. 
normal. However, step lengths between both groups when walking with the PGO were similar even though both were significantly smaller than normal healthy participant walking $(P=0.001$ and 0.007 ) for persons with SCI and healthy participants, respectively.

Hip ROM when walking with the PGO was $10^{\circ}$ less for persons with SCI than that demonstrated by healthy participants walking with the PGO, whereas knee ROM was only reduced by $4^{\circ}$. It would appear that the two main factors in producing the demonstrated reduction in persons with SCI walking speed with the PGO were the reduction in hip ROM and step length when compared with that demonstrated by healthy participants walking with the orthosis. The walking speed was less in healthy participants walking with PGO compared with normal walking (Table 4). Although there was no significant difference in hip joint ROM between healthy participants walking with PGO compared with normal walking, the mean of this parameter reduced $12 \%$ between the two test groups. Also, when considering step length, there was significant difference in this parameter between healthy participants walking with the PGO compared with normal walking. The step length decreased by a mean of $20 \%$ between the test groups. To find out the causes of lower speed, variables such as stance and swing time, and segmental velocity/acceleration need to be investigated. Factors such as mechanical resistance of the joints in the PGO may also provide useful information. Reduction of gait determinants measured may have been related to postural stabilization, as well as inefficiency of mechanical energy transfer between the lower limbs by the orthosis. Further studies in this field are needed to analyze these effects.

The mean ankle joint ROM decreased significantly as compared with normal walking, but there was no significant difference between walking with the PGO by healthy participants and persons with SCI. The use of a solid ankle foot orthosis in the new PGO caused these limitations to ankle ROM because of the limited joint movement allowed within the ankle foot orthosis superstructures. In comparison, joint angles between healthy participants and persons with SCI demonstrated significant differences between them in hip and knee joint ROM. However, the means of hip and knee joint ROM with this new PGO on SCI patients was improved when compared with previous studies in this field. ${ }^{13,14}$

The evidence from this study would also suggest that the two main factors in producing the demonstrated reduction in persons with SCI walking speed with the PGO were the reduction in hip ROM and cadence when compared with that demonstrated by healthy participants walking with the orthosis. This study therefore was effective in identifying specific parameters that would need to be improved in further development of the device. Practical issues such as user acceptance, ease of donning and doffing, cosmesis, energy cost and clinical utility, such as climbing stairs, are also important design considerations for future development of the device.

When compared with the previous studies in this field, the results of this study in evaluation of the effect of the PGO on walking in persons with SCI showed faster gait speeds and improved step lengths. The walking speed and step length demonstrated in walking with powered orthoses in previous studies ${ }^{1,12,15}$ were all slower and shorter, respectively, than that shown in this study. Walking speeds previously reported during walking with the IRGO and $\mathrm{ARGO}^{4,16,17}$ were slower than that shown in this study. Participants in this study initially had a slower walking speed, but this improved after gait training with the PGO as they had acquired walking skills with the new orthosis and thus they had improvement in their speed of walking and step length when walking with powered orthosis during the walking trials. Significant differences were demonstrated between walking with new PGO on healthy participants and persons with SCI. Using a powered mechanism in the ankle joint or a movable ankle joint or using separate electric actuators in the hip joints may improve parameters to more closely approximate them to the optimal level of walking with this type of orthosis. ${ }^{4,16,17}$

Future design constraints in terms of challenges to postural stability and over-reliance on upper limb forces in bipedal, tripedal and quadripedal gait patterns with increased stride length and/or cadence need to be considered in future orthotic designs. For example, it cannot be assumed that proportionate increases in stride length and cadence can be achieved in a manner similar to able-bodied individuals by people with SCI, as both intrinsic and extrinsic stability needs to be maintained when walking with increased stride length and cadence.

\section{Limitations}

There were some limitations in this study. According to lack of power and study design and the small sample size, a larger study with more participants will be needed to confirm the results of this study. In addition, the findings of this study may not be applicable for other orthotic devices until this larger study is performed. The evaluation of the effect of this PGO on loading on upper limb joints, performance of activities of daily living and its equivalency or superiority to currently available mechanical orthoses (e.g. the IRGO or ARGO) will therefore need to be determined when used by participants with paraplegia.

\section{CONCLUSION}

The results of this study demonstrated that walking with a PGO by healthy participants still significantly reduced critical gait parameters, and further development work is therefore needed to produce a more effective device to more closely match the gait parameters of normal walking by healthy subjects.

\section{DATA ARCHIVING}

There were no data to deposit.

\section{CONFLICT OF INTEREST}

The authors declare no conflict of interest.

1 Arazpour M, Bani MA, Hutchins SW. Reciprocal gait orthoses and powered gait orthoses for walking by spinal cord injury patients. Prosth Orth Int 2013; 37: 14-21.

2 Arazpour M, Bani M, Hutchins S, Jones R. The physiological cost index of walking with mechanical and powered gait orthosis in patients with spinal cord injury. Spinal Cord 2013; 51: 356-359.

3 Bernardi M, Canale I, Castellano V, Di Filippo L, Felici F, Marchetti M. The efficiency of walking of paraplegic patients using a reciprocating gait orthosis. Spinal Cord 1995; 33: 409-415.

4 Massucci M, Brunetti G, Piperno R, Betti L, Franceschini M. Walking with the advanced reciprocating gait orthosis (ARGO) in thoracic paraplegic patients: energy expenditure and cardiorespiratory performance. Spinal Cord 1998; 36: 223-227.

5 Yang L, Condie D, Granat M, Paul J, Rowley D. Effects of joint motion constraints on the gait of normal subjects and their implications on the further development of hybrid FES orthosis for paraplegic persons. J Biomech 1996; 29: 217-226.

6 Dall P, Müller B, Stallard I, Edwards J, Granat M. The functional use of the reciprocal hip mechanism during gait for paraplegic patients walking in the Louisiana State University reciprocating gait orthosis. Prosth Orth Int 1999; 23: 152-162.

7 Hirokawa S, Grimm M, Solomonow M, Baratta R, Shoji H, D'ambrosia R. Energy consumption in paraplegic ambulation using the reciprocating gait orthosis and electric stimulation of the thigh muscles. Arch Phys Med Rehab 1990; 71: 687.

8 Kawashima N, Taguchi D, Nakazawa K, Akai M. Effect of lesion level on the orthotic gait performance in individuals with complete paraplegia. Spinal Cord 2006; 44: 487-494.

9 Audu ML, To CS, Kobetic R, Triolo RJ. Gait evaluation of a novel hip constraint orthosis with implication for walking in paraplegia. IEEE Trans Neural Syst Rehab Eng 2010; 18: 610-618. 
10 Arazpour M, Chitsazan A, Hutchins SW, Ghomshe FT, Mousavi ME, Takamjani EE et al. Design and simulation of a new powered gait orthosis for paraplegic patients. Prosth Orth Int 2012; 36: 125-130.

11 Arazpour M, Chitsazan A, Hutchins SW, Mousavi ME, Takamjani EE, Ghomshe FT et al. Evaluation of a novel powered gait orthosis for walking by a spinal cord injury patient. Prosth Orth Int 2012; 36: 239-246.

12 Arazpour M, Chitsazan A, Hutchins SW, Ghomshe FT, Mousavi ME, Takamjani EE et al. Evaluation of a novel powered hip orthosis for walking by a spinal cord injury patient: a single case study. Prosth Orth Int 2012; 36: 105-112.

13 Rasmussen AA, Smith KM, Damiano DL. Biomechanical evaluation of the combination of bilateral stance-control knee-ankle-foot orthoses and a reciprocating gait orthosis in an adult with a spinal cord injury. J Prosth Ortho 2007; 19: 42.
14 Johnson WB, Fatone S, Gard SA. Walking mechanics of persons who use reciprocating gait orthoses. J Rehab Res Dev 2009; 46: 435-446.

15 Kang S, Ryu J, Moon I, Kim K and Mun M (eds). Walker Gait Analysis of Powered Gait Orthosis for Paraplegic. World Congress on Medical Physics and Biomedical Engineering 2006. Springer: Berlin, 2007.

16 Leung AKL, Wong AFY, Wong ECW, Hutchins SW. The physiological cost index of walking with an isocentric reciprocating gait orthosis among patients with T12-L1 spinal cord injury. Prosth Orth Int 2009; 33: 61-68.

17 ljzerman MJ, Baardman G, van't Hof MA, Boom HB, Hermens HJ, Veltink PH. Validity and reproducibility of crutch force and heart rate measurements to assess energy expenditure of paraplegic gait. Arch Phys Med Rehab 1999; 80 1017-1023. 